\title{
Nonlinear MHD Flow and Heat Transfer over a Power- Law Stretching Plate with Free Stream Pressure Gradient and Viscous Dissipation in Presence of Variable Thermal Diffusivity
}

\author{
M. Thiagarajan ${ }^{\dagger}$ and A. S. Sangeetha ${ }^{\dagger \dagger}$ \\ $\dagger$ Department of Mathematics, PSG College of Arts and Science \\ Coimbatore-641 014. INDIA \\ Email: thiyagu26@ rediffmail.com \\ $\dagger \dagger$ Department of Mathematics, Park College of Engineering and Technology \\ Coimbatore-641 659. INDIA \\ Email: sangu.dakshin@gmail.com
}

\begin{abstract}
The numerical analysis is carried out on steady nonlinear magnetohydrodynamic flow and heat transfer over a power-law stretching plate with free stream pressure gradient and viscous dissipation in the presence of variable thermal diffusivity and variable transverse magnetic field. The thermal diffusivity is assumed to vary as linear functions of temperature. Using similarity transformation, the nonlinear partial differential equations are converted into nonlinear ordinary differential equations. Numerical solutions of these equations are obtained with the help of Runge-Kutta shooting method. The effects of magnetic field, stretching parameter, Prandtl number, free stream velocity ratio, Eckert number and thermal diffusivity parameter on skin friction, velocity, rate of heat transfer and temperature are thoroughly discussed. It is found that the effect of magnetic field is to decrease the velocity and enhance the temperature.
\end{abstract}

Keywords: Magnetohydrodynamics, Free stream pressure gradient, Prandtl number, Thermal diffusivity, Viscous dissipation.

\section{Nomenclature}

$U_{\infty}(x)$ - Free stream velocity

$U_{w}(x)$ - Stretching plate velocity

$U(x)$ - Composite reference velocity

$\delta$ - Boundary layer thickness

$\sigma \quad$ - Electrical conductivity 


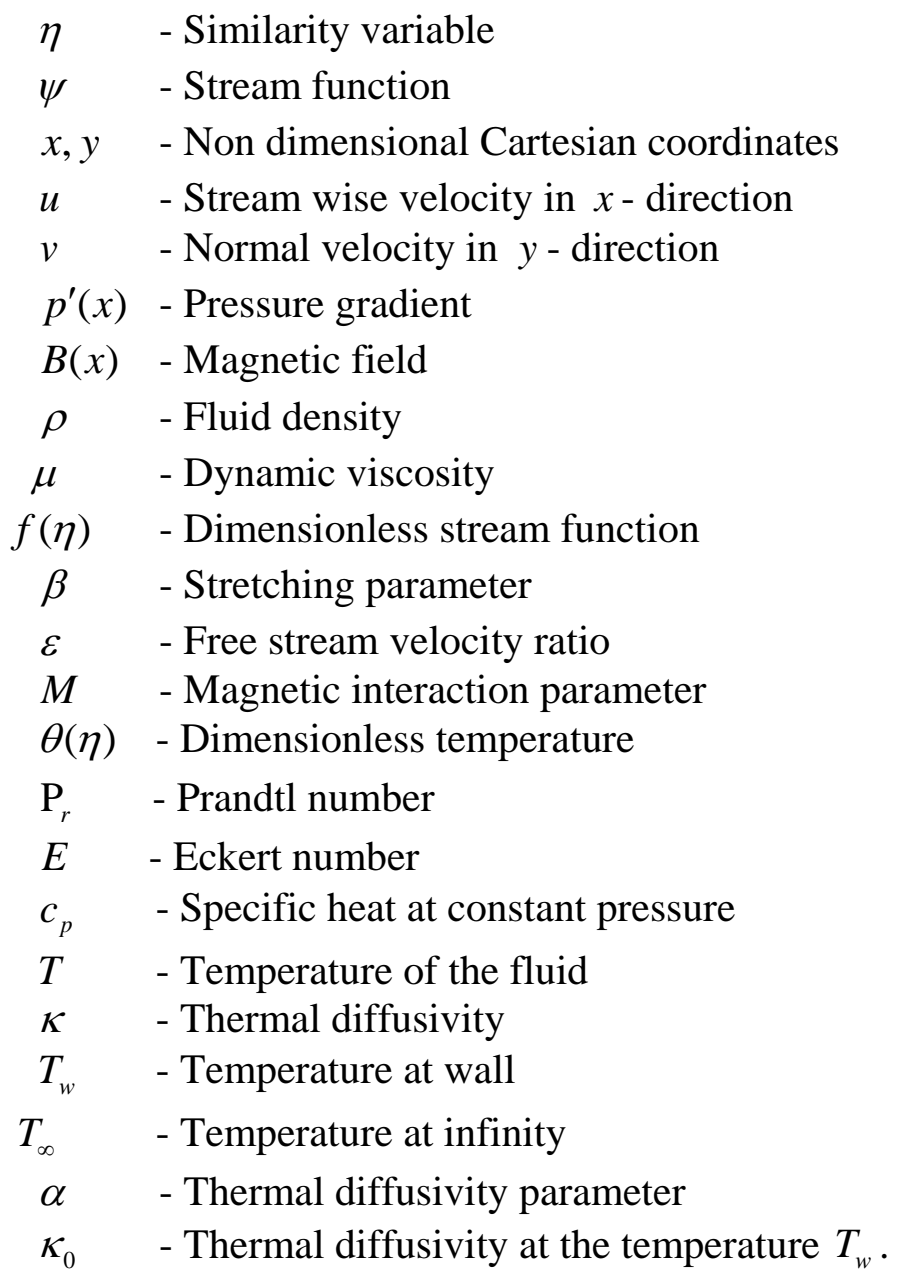

\section{Introduction}

Boundary layer flows on a stretching surface occur in several engineering applications. Aerodynamic extrusion of a plastic sheet, the cooling of an infinite metallic plate in a cooling bath, condensation processes and geophysical and industrial fields, are examples of practical applications. When the effects of the magnetic and fluid forces are considered together, the resulting boundary layer equations become intractable for an analytical treatment. Idealized models have therefore been investigated in literature with a view to understanding the individual as well as coupled effects of the flow parameters Pai [1] and Schlichting [2].

Sakiadis [3, 4] was the first to investigate the boundary layer flow past a moving solid surface of a viscous fluid with a constant velocity. Later, the numerical results of Sakiadis $[3,4]$ were confirmed by Tsou, Sparrow and Goldstain [5] analytically and experimentally. The steady laminar boundary layer flow over a continuously stretching plate was first studied by Crane [6], who obtained an exact solution to the Navier-Stokes equations. Many investigators have extended Crane's work to study flow, heat and/or mass transfer under different physical situations. The development of the boundary layer due to a stretching permeable sheet was studied by Gupta and Gupta [7], who reported an exact solution for the flow field and a solution in incomplete gamma functions for the 
thermal field. Ali [8] studied the general case when the sheet is stretched with stretching velocity of the form $x^{m}$.

The heat transfer aspect for the problem posed by Crane [6] was studied by Grubka and Bobba [9], who reported the solution for the energy equation interms of Kummer's functions. Chen and Char [10] investigated the effects of suction and injection on the heat transfer characteristics of a continuous, linearly stretching sheet for both the power-law surface temperature and the power-law surface heat flux variations. Recently, the similarity solution of magnetohydrodynamic boundary layer driven by the stretching surface boundary and pressure gradient, each proportional to the same power-law of the downstream coordinate based on composite reference velocity (sum of the velocities of stretching boundary and free stream) has been examined by Thiagarajan and Sangeetha [11].

Most of the previous studies were concerned with magnetohydrodynamic flow. Dandapat, Santra and Vajravelu [12] investigated the effect of variable fluid properties and thermo capillarity on the flow of a thin film on an unsteady stretching sheet. Chiam [13] considered the effect of a variable thermal conductivity on the flow and heat transfer from a linearly stretching sheet. Viscous dissipation changes the temperature distributions by playing a role like an energy source, which leads to affected heat transfer rates. The merit of the effect of viscous dissipation depends on whether the plate is being cooled or heated.

Hence the present study investigates the effect of viscous dissipation and variable thermal diffusivity on nonlinear MHD flow over a heated stretching plate with free stream pressure gradient.

\section{Formulation of the Problem}

Consider the steady two-dimensional laminar boundary layer flow of a viscous incompressible electrically conducting fluid over a heated stretching plate with free stream pressure gradient in the presence of a transverse variable magnetic field $B(x)$ and variable thermal diffusivity. The effect of viscous dissipation is considered. The continuity, momentum and energy equations governing such type of flow are written as

$$
\begin{aligned}
& \frac{\partial u}{\partial x}+\frac{\partial v}{\partial y}=0 \\
& \rho\left(u \frac{\partial u}{\partial x}+v \frac{\partial u}{\partial y}\right)=-p^{\prime}(x)+\mu \frac{\partial^{2} u}{\partial y^{2}}-\sigma B^{2}(x) u, p^{\prime}(x)=-\rho U_{\infty} \frac{d U_{\infty}}{d x}-\sigma B^{2}(x) U_{\infty} \\
& \rho c_{p}\left(u \frac{\partial T}{\partial x}+v \frac{\partial T}{\partial y}\right)=\frac{\partial}{\partial y}\left(\kappa \frac{\partial T}{\partial y}\right)+\mu\left(\frac{\partial u}{\partial y}\right)^{2}
\end{aligned}
$$

The relevant boundary conditions are

$$
u=U_{w}(x), v=0, T=T_{w} \text { at } y=0 ; T \rightarrow T_{\infty}, u \rightarrow U_{\infty}(x) \text { as } \frac{y}{\delta} \rightarrow \infty
$$


where $u$ is the stream wise velocity in $x$-direction and $v$ is the normal velocity in $y$ direction, $p^{\prime}(x)$ is the pressure gradient, $\rho$ is fluid density, $\mu$ is coefficient of viscosity, $\sigma$ is electrical conductivity, $c_{p}$ is specific heat at constant pressure, $\kappa$ is thermal diffusivity, $T$ is temperature of the fluid and $B(x)$ is the magnetic field, $U_{w}(x)$ is the stretching plate velocity, $U_{\infty}(x)$ is the free stream velocity, $T_{w}$ is wall temperature and $T_{\infty}$ is temperature of the fluid at infinity.

In writing the above equations, the induced magnetic field is assumed to be negligible. Further, the external electrical field is assumed to be zero and the electric field due to polarization of charges is also negligible.

The similarity solution exists if stretching plate velocity $U_{w}(x)$ and velocity at the edge of boundary layer $U_{\infty}(x)$ obey the following power-law relations:

$U_{w}=U_{0 w} x^{m}, \quad U_{\infty}=U_{0 \infty} x^{m}$

Utilizing similarity variables based on the composite reference velocity $U(x)$ following Afzal, Baderuddin and Elgarvi [14]

$$
\begin{aligned}
& \eta(x, y)=y \sqrt{\frac{(1+m) U(x)}{2 v x}} \\
& \psi(x, y)=\sqrt{\frac{2 v x U(x)}{1+m}} f(\eta)
\end{aligned}
$$

and defining

$$
\begin{aligned}
& u=\frac{\partial \psi}{\partial y} ; \quad v=-\frac{\partial \psi}{\partial x} \\
& U(x)=U_{\infty}(x)+U_{w}(x) \\
& \theta(\eta)=\frac{T-T_{\infty}}{T_{w}-T_{\infty}}
\end{aligned}
$$

where $\eta$ is the similarity variable, $f(\eta)$ is the dimensionless stream function and $\theta(\eta)$ is the dimensionless temperature, the momentum and energy equations can be transformed into nonlinear ordinary differential equations. The continuity equation (1) is automatically satisfied.

The variation of thermal diffusivity with the dimensionless temperature is written as

$$
\kappa=\kappa_{0}(1+\alpha \theta)
$$

where $\alpha$ is a parameter which depends on the nature of the fluid, $\kappa_{0}$ is the value of thermal diffusivity at the temperature $T_{w}$. 
The special form for magnetic field $B(x)=B_{0} x^{\frac{m-1}{2}}$ is chosen to obtain the similarity solution.

Using the relations (5) to (11) in Eqs. (2) to (4), we get

$$
\begin{aligned}
& f^{\prime \prime \prime}+f f^{\prime \prime}-\beta\left(f^{\prime 2}-\varepsilon^{2}\right)-M\left(f^{\prime}-\varepsilon\right)=0 \\
& (1+\alpha \theta) \theta^{\prime \prime}+\alpha \theta^{\prime^{2}}+\operatorname{Pr}\left(f \theta^{\prime}+E f^{\prime^{2}}\right)=0
\end{aligned}
$$

The corresponding boundary conditions are

$$
\begin{aligned}
& f(0)=0, f^{\prime}(0)=1-\varepsilon, f^{\prime}(\infty)=\varepsilon \\
& \theta(0)=1, \theta(\infty)=0
\end{aligned}
$$

where $M=\frac{2 \sigma B_{0}{ }^{2}}{\rho\left(U_{0 \infty}+U_{0 w}\right)(1+m)}$ is the magnetic interaction parameter,

$$
\begin{aligned}
& \beta=\frac{2 m}{m+1} \text { is the stretching parameter, } \\
& \varepsilon=\frac{U_{0 \infty}}{U_{0 \infty}+U_{0 w}} \text { is the free stream velocity ratio } \\
& \operatorname{Pr}=\frac{\mu c_{p}}{\kappa_{0}} \text { is the Prandtl number } \\
& E=\frac{U^{2}}{c_{p}\left(T_{w}-T_{\infty}\right)} \text { is the Eckert number }
\end{aligned}
$$

and $\alpha$ is the thermal diffusivity parameter.

Eqs. (12) and (14) for $\varepsilon=1$ and $M=0$ are classical Falkner-Skan equations and for $\varepsilon=0$ and $M=0$ are the equations for stretching sheet proposed by Afzal and Varshney [15] and Afzal [16, 17].

In the absence of magnetic field, equation (12) reduces to that of Afzal [18].

\section{Numerical Solution of the Problem}

Eqs. (12) and (13) subject to boundary conditions (14) and (15) are nonlinear ordinary differential equations which constitute the nonlinear boundary value problem and are solved numerically using Runge-Kutta shooting method. The crux of the problem is that we have to make an initial guess for the values of $f^{\prime \prime}(0)$ and $\theta^{\prime}(0)$ to initiate the shooting process. The success of the procedure depends very much on how good this guess is. Numerical results are obtained for several values of the physical parameters $M$, $\beta, \operatorname{Pr}, \varepsilon, E$ and $\alpha$. 


\section{Results and Discussion}

The numerical solutions of nonlinear MHD flow and heat transfer over a power-law stretching plate with free stream pressure gradient and viscous dissipation in the presence of variable thermal diffusivity has been obtained by Runge-Kutta shooting method by fixing several values for the physical parameters. Numerical results are depicted graphically by means of figures for dimensionless velocity $f^{\prime}(\eta)$, dimensionless temperature $\theta(\eta)$, skin friction $f^{\prime \prime}(0)$ and the rate of heat transfer $\theta^{\prime}(0)$ for several sets of values of magnetic interaction parameter $M$, stretching parameter $\beta$, Prandtl number $\operatorname{Pr}$, free stream velocity ratio $\varepsilon$, Eckert number $E$ and thermal diffusivity parameter $\alpha$. Fig. 1 displays the plot of velocity profile $f^{\prime}(\eta)$ against $\eta$ with non-dimensional parameter $0 \leq \varepsilon \leq 1$ for each value of stretching parameter $\beta=0.0$ to 2.0 when $M=0.2, E=0.01, \alpha=1.0$ and $\operatorname{Pr}=7.0$. For each value $\beta$ the velocity profile shows gradual variation as velocity ratio $\varepsilon$ increased from 0 to 1 . The gradient $f^{\prime}(\eta)$ is positive for $\varepsilon>\frac{1}{2}$ and negative for $\varepsilon<\frac{1}{2}$.

Fig. 2 demonstrates the dimensionless temperature $\theta(\eta)$ against $\eta$ with non-dimensional parameter $0 \leq \varepsilon \leq 1$ for each value of stretching parameter $\beta=0.0$ to 2.0 when $M=0.2, E=0.01, \alpha=1.0$ and $\operatorname{Pr}=7.0$. It is observed that as free stream velocity ratio $\varepsilon$ increases, temperature $\theta(\eta)$ increases which physically conveys the fact that the effect of free stream velocity ratio is to enhance the temperature.

Fig. 3 represents the influence of magnetic interaction parameter $M$ over the nondimensional velocity $f^{\prime}(\eta)$ when $\beta=1.0$. It is seen that as magnetic interaction parameter $M$ increases, velocity $f^{\prime}(\eta)$ decreases elucidating the fact that the effect of magnetic field is to decelerate the velocity.

Fig. 4 gives the dimensionless temperature for different values of magnetic interaction parameter $M$. It is noticed that for increasing values of magnetic interaction parameter $M$, the temperature $\theta(\eta)$ increases which physically conveys the fact that the effect of magnetic field is to enhance the temperature. Also it is interesting to see that the boundary layer becomes thicker due to the effect of increasing magnetic field.

Variation in dimensionless velocity $f^{\prime}(\eta)$ due to the stretching parameter $\beta$ is visualized through Fig. 5. As the stretching parameter $\beta$ increases, velocity $f^{\prime}(\eta)$ increases disclosing the fact that the effect of stretching of the wall is to accelerate the velocity.

Non-dimensional temperature $\theta(\eta)$ for different values of the stretching parameter $\beta$ is presented graphically in Fig. 6. It is seen that the effect of stretching parameter is to reduce the temperature, as it increases and also to reduce the thickness of thermomagnetic layer.

The temperature profile $\theta(\eta)$ against $\eta$ for different values of $E$ is shown in Fig. 7. It is noteworthy that the temperature profiles are closer to each other due to the effect of Eckert number $E$.

The effects of thermal diffusivity parameter $\alpha$ on temperature field is given in Fig. 8. It is observed that the fluid temperature increases with the increasing values of $\alpha$. 
For $\beta$ positive, and $0 \leq \varepsilon \leq 1$ the numerical solutions $f^{\prime \prime}(0)$ and $\theta^{\prime}(0)$ against $\varepsilon$ with parameter $\beta=0.0$ to 2.0 are illustrated in Figs. 9 to 12. It is noted that the skin friction $f^{\prime \prime}(0)$ and rate of heat transfer $\theta^{\prime}(0)$ are unique against the free stream velocity ratio $\varepsilon$.

Fig. 13 gives clearly the effect of magnetic interaction parameter $M$ over the skin friction $f^{\prime \prime}(0)$ due to stretching parameter $\beta$. The skin friction is increased due to the effect of magnetic interaction parameter $M$ whereas it is enhanced due to the effect of stretching parameter $\beta$.

Fig. 14 gives clearly the effect of magnetic interaction parameter $M$ over the rate of heat transfer $\theta^{\prime}(0)$ due to stretching parameter $\beta$. The rate of heat transfer is decreased due to the effect of magnetic interaction parameter $M$ whereas it is suppressed due to the effect of stretching parameter $\beta$.

Fig. 15 gives clearly the effect of Prandtl number Pr over the rate of heat transfer $\theta^{\prime}(0)$ due to free stream velocity ratio $\varepsilon$. The rate of heat transfer is decreased due to the effect of Prandtl number whereas it is enhanced due to the effect of free stream velocity ratio.

\section{Conclusion}

- Dimensionless velocity is reduced due to the influence of magnetic field where its effect is to increase the stretching parameter.

- The gradual variation in dimensionless velocity as increasing free stream velocity.

- The effect of magnetic field is to enhance the temperature.

- The effect of stretching parameter is to reduce the temperature and also to reduce the thickness of thermomagnetic layer.

- The effect of stretching parameter is increase the skin friction and decrease the rate of heat transfer.

- Skin friction and rate of heat transfer enhance as increasing free stream velocity.

- The rate of heat transfer is suppressed due to the effect of Prandtl number.

- Fluid temperature increases with increasing thermal diffusivity parameter.

- The temperature in the boundary layer slightly increases due to viscous dissipation. It is hoped that with the help of our present model, the physics of flow over the stretching sheet may be utilized as the basis of many engineering and scientific applications. The results derived from the present study may be useful for different model investigations.

\section{References}

[1] S.I. Pai, Magnetogasdynamics and Plasma Dynamics, Springer Bertin 1962.

[2] H.Schlichting, Boundary Layer Theory, McGraw-Hill, New York 1979.

[3] B.C. Sakiadis, Boundary-layer behavior on continuous solid surfaces: I. Boundarylayer equations for two-dimensional and axisymmetric flow, AIChE J. 7 (1961) 2628.

[4] B.C. Sakiadis, Boundary-layer behavior on continuous solid surfaces: II. The boundary layer on a continuous flat surface, AIChE J. 7 (1961) 221-225. 
[5] F.K. Tsou, E.M. Sparrow, R.J. Goldstain, Flow and heat transfer in the boundary layer on a continuous moving surface, Int. J. Heat Mass Transfer 1 (1967) 219-235.

[6] L.J. Crane, Flow past a stretching plate, Z. Angew. Math. Phys. (ZAMP) 21 (1970) 645-647.

[7] P.S. Gupta, A.S. Gupta, Heat and mass transfer on a stretching sheet with suction or blowing, Can. J. Chem. Eng. 55 (1977) 744-746.

[8] M.E. Ali, On thermal boundary layer on a power-law stretched surface with suction or injection, Int. J. Heat Fluid Flow 16 (1995) 280-290.

[9] L.J. Grubka, K.M. Bobba, Heat transfer characteristics of a continuous, stretching surface with variable temperature, J. Heat transfer 107 (1985) 248-250.

[10] C.K. Chen, M.I. Char, Heat transfer of a continuous, stretching surface with suction or blowing, J. Mathematical Anal. Appl. 135 (1988) 568-580.

[11] M. Thiagarajan, A.S. Sangeetha, Magnetohydrodynamic flow over a power-law stretching plate with free stream pressure gradient, JEHMT 32 (2010) 375-384.

[12] B.S. Dandapat, B. Santra, K. Vajravelu, The effects of variable fluid properties and thermocapilliarity on the flow of a thin film on an unsteady stretching sheet, Int. J. Heat Mass Transfer 50 (2007) 991-996.

[13] T.C. Chiam, Heat transfer in a fluid with variable thermal conductivity over a linearly stretching sheet, Acta Mech. 129 (1998) 63-72.

[14] N. Afzal, A. Baderuddin, A.A. Elgarvi, Momentum and heat transport on a continuous flat surface moving in a parallel stream, Int. J. Heat Mass Transfer 36 (1993) 3399- 3403.

[15] N. Afzal, I.S. Varshney, The cooling of a low heat resistance sheet moving through a fluid, Warme Stoffubertrag 14 (1980) 289-293.

[16] N. Afzal, The cooling of a low heat resistance sheet moving through a fluid: a rejoinder, Warme Stoffubertrag 17 (1983) 217-219.

[17] N. Afzal, Heat transfer from a stretching surface, Int. J. Heat Mass Transfer 36 (1993) 1128-1131.

[18] N. Afzal, Momentum transfer on power-law stretching plate with free stream pressure gradient, Int. J. Eng. Sci. 41 (2003) 1197-1207. 

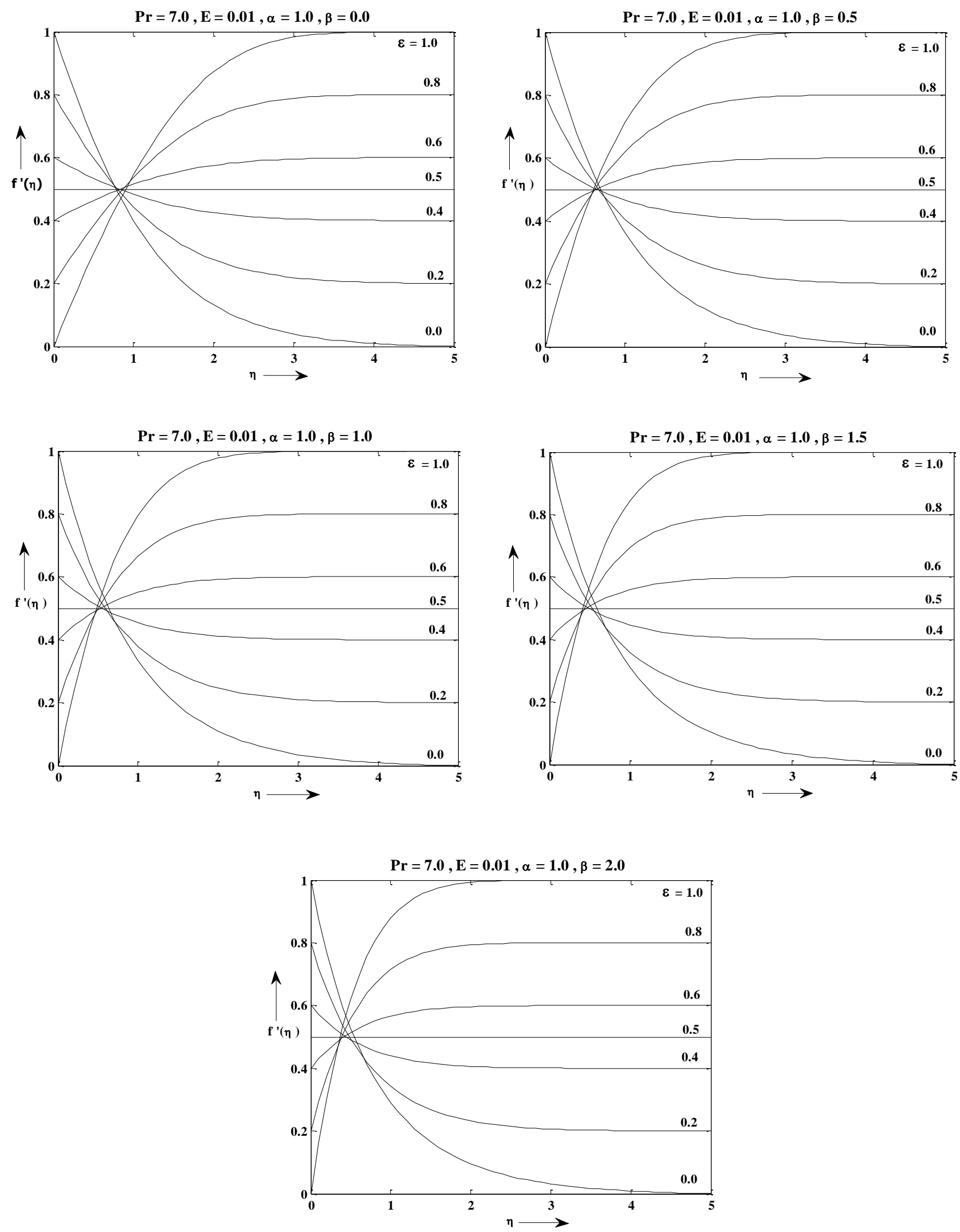

Fig. 1 The velocity distribution $f^{\prime}(\eta)$ against $\eta$ for free stream velocity ratio $0 \leq \varepsilon \leq 1$ for $\beta=0.0$ to 2.0 when $M=0.2$. 

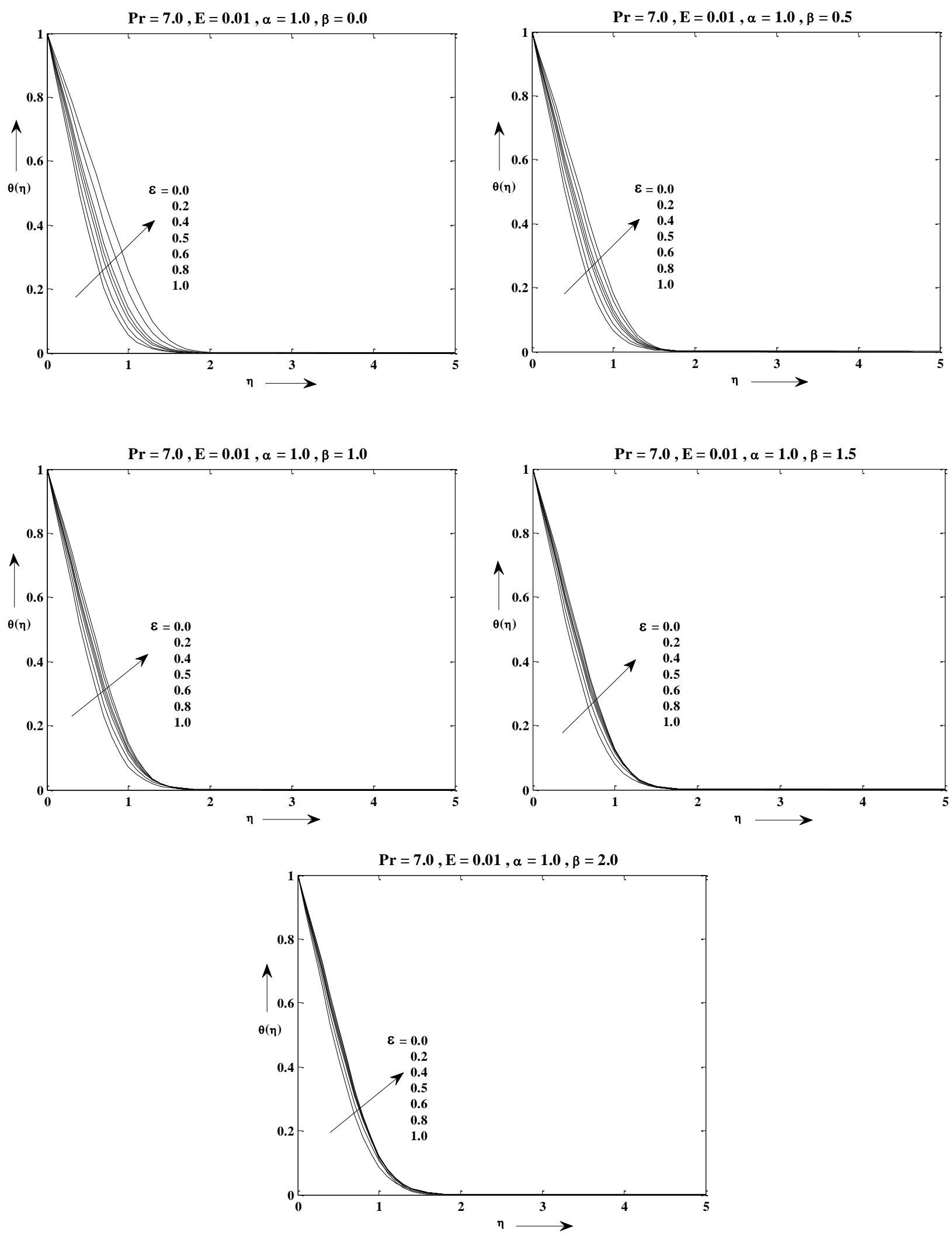

Fig. 2 The temperature distribution $\theta(\eta)$ against $\eta$ for free stream velocity ratio $0 \leq \varepsilon \leq 1$ for $\beta=0.0$ to 2.0 when $M=0.2$. 


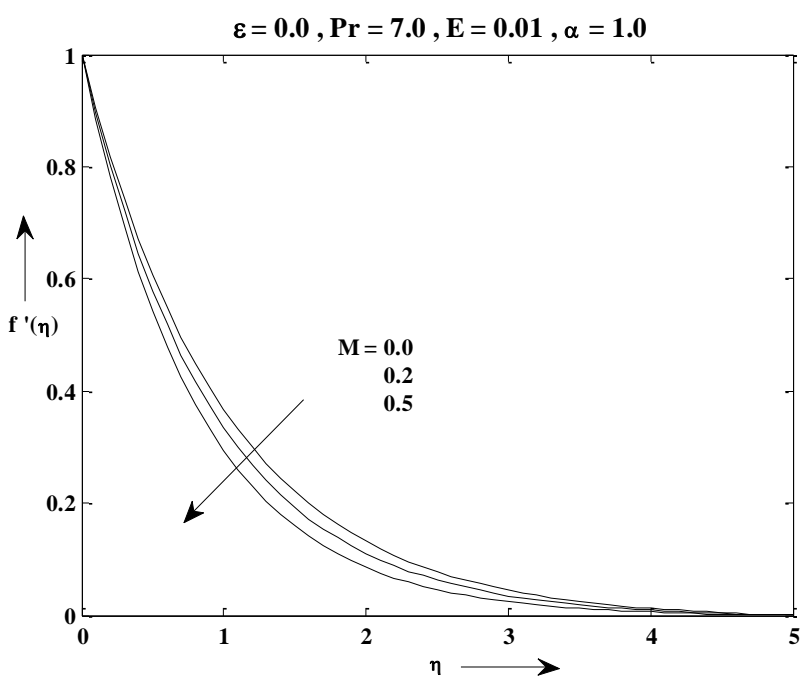

Fig. 3 The velocity distribution $f^{\prime}(\eta)$ against $\eta$ for magnetic interaction parameter $M$ when $\beta=1.0$.

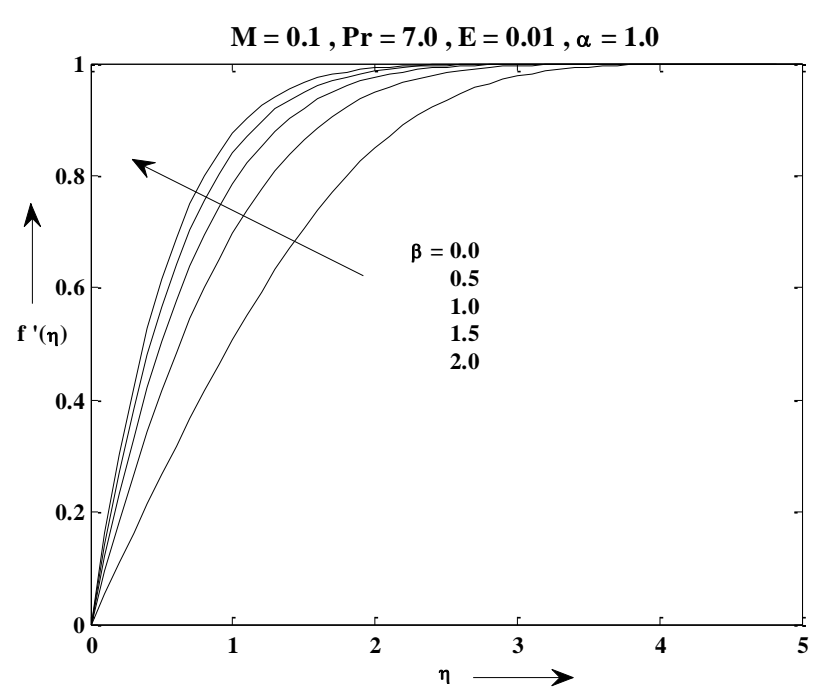

Fig. 5 The velocity distribution $f^{\prime}(\eta)$ against $\eta$ for stretching parameter $\beta$ when $\varepsilon=1.0$.

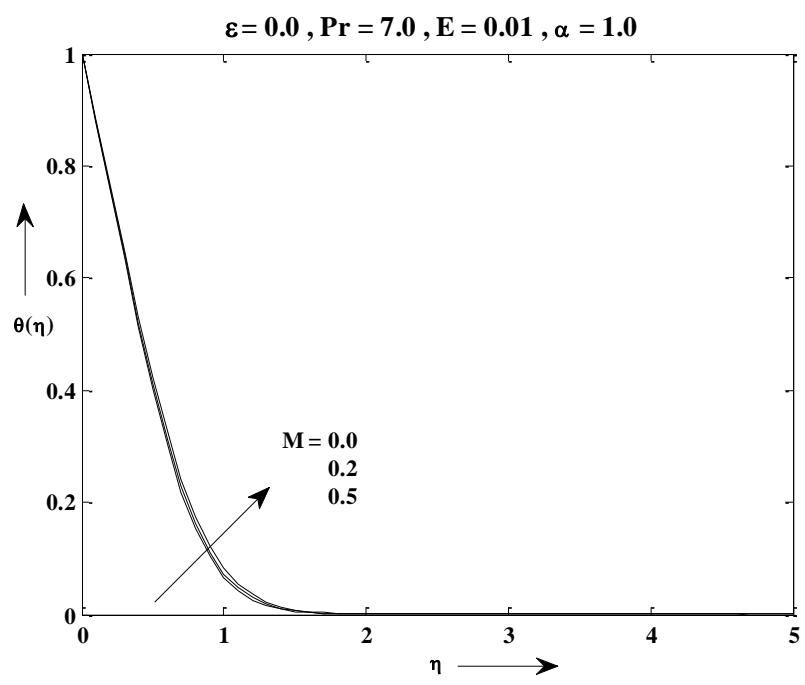

Fig. 4 The temperature distribution $\theta(\eta)$ against $\eta$ for magnetic interaction parameter $M$ when $\beta=1.0$.

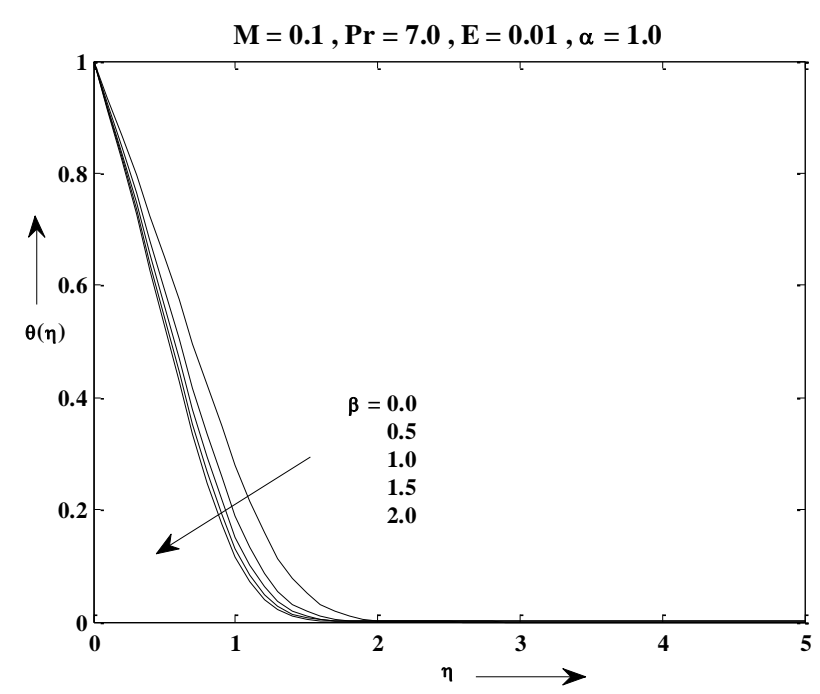

Fig. 6 The temperature distribution $\theta(\eta)$ against $\eta$ for stretching parameter $\beta$ when $\varepsilon=1.0$. 


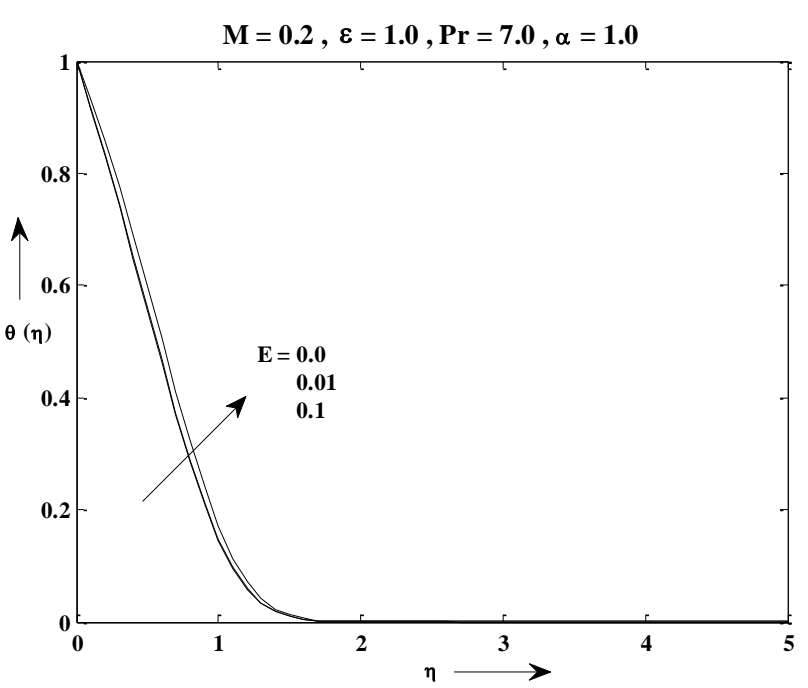

Fig. 7 The temperature distribution $\theta(\eta)$ against $\eta$ for Eckert number $E$ when $\beta=1.0$.

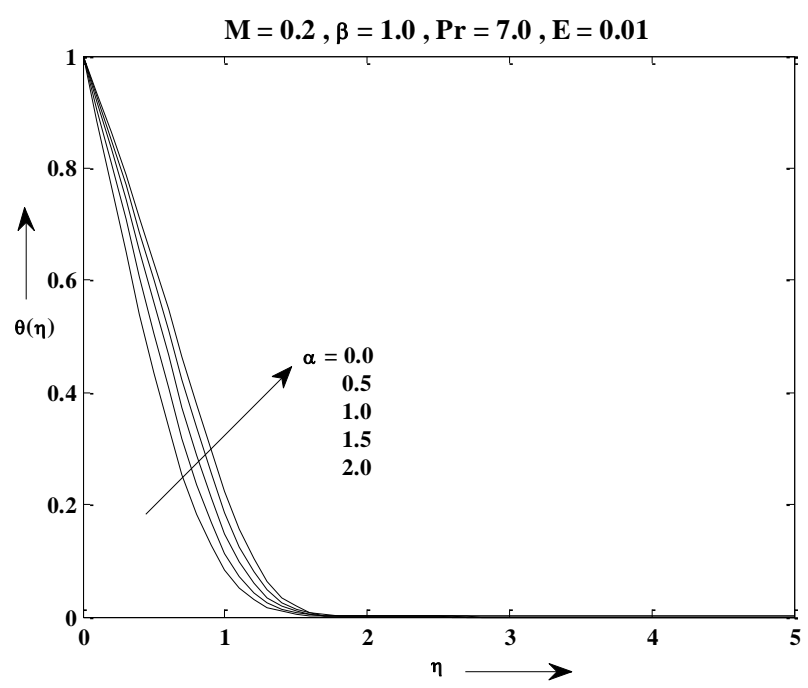

Fig. 8 The temperature distribution $\theta(\eta)$ against $\eta$ for thermal diffusivity parameter $\alpha$ when $\varepsilon=1.0$.

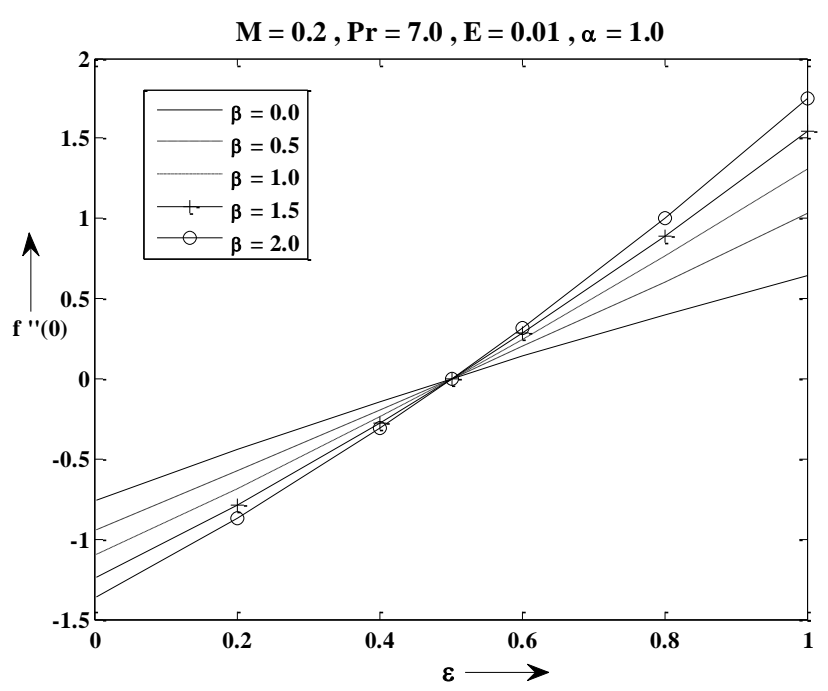

Fig. 9 Skin friction for different $\beta$.

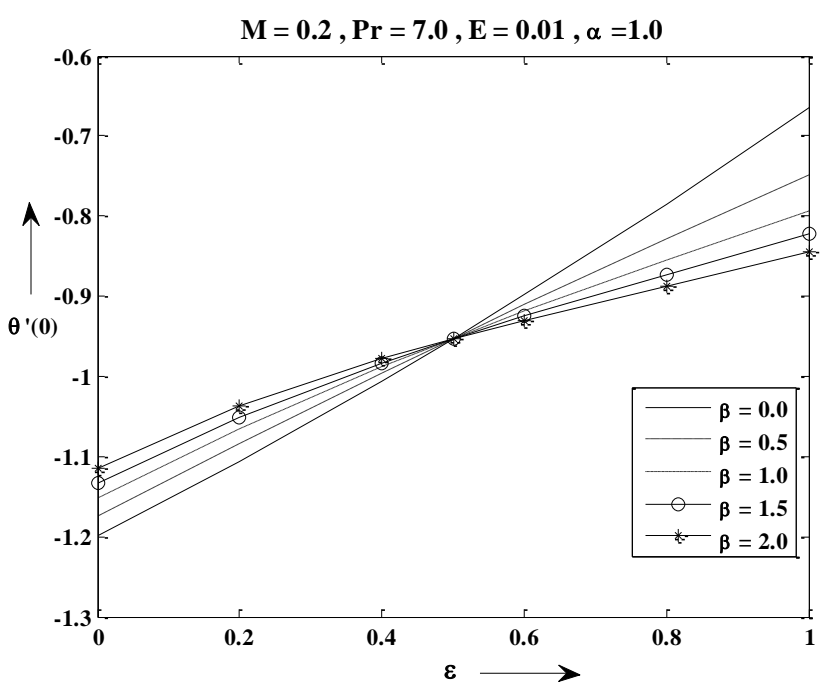

Fig. 10 The rate of heat transfer for different $\beta$. 


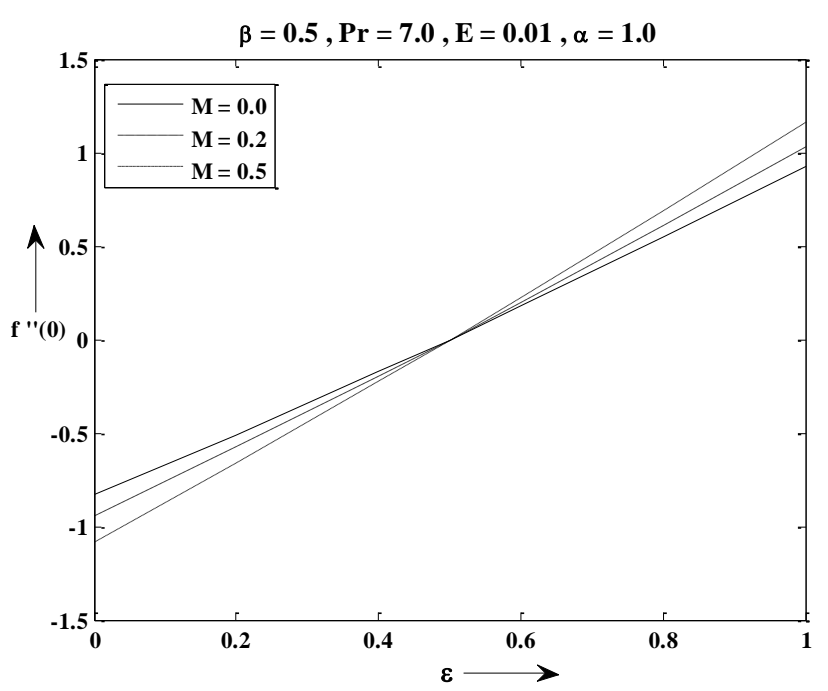

Fig. 11 Skin friction for different $M$.

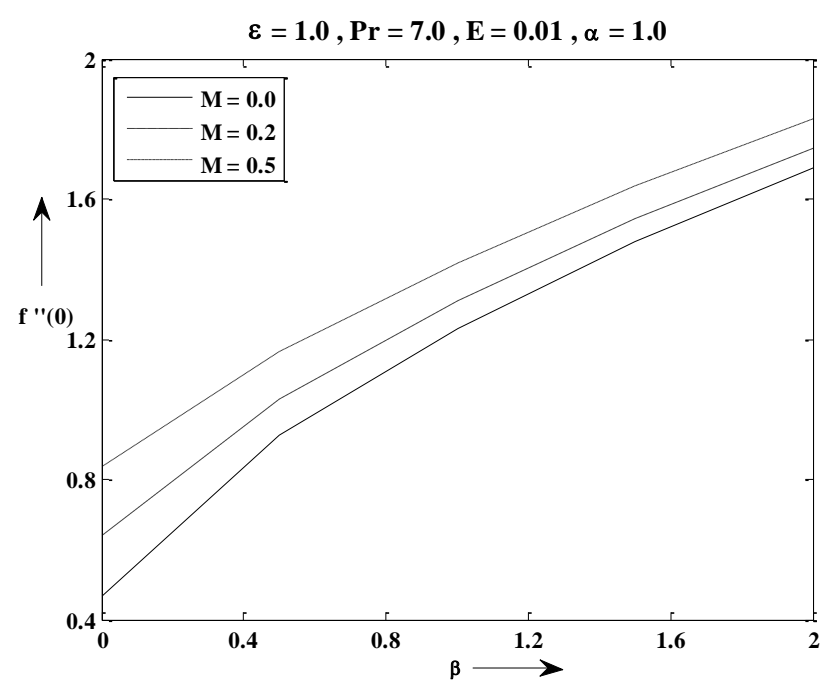

Fig. 13 Skin friction against $\beta$ for different $M$.

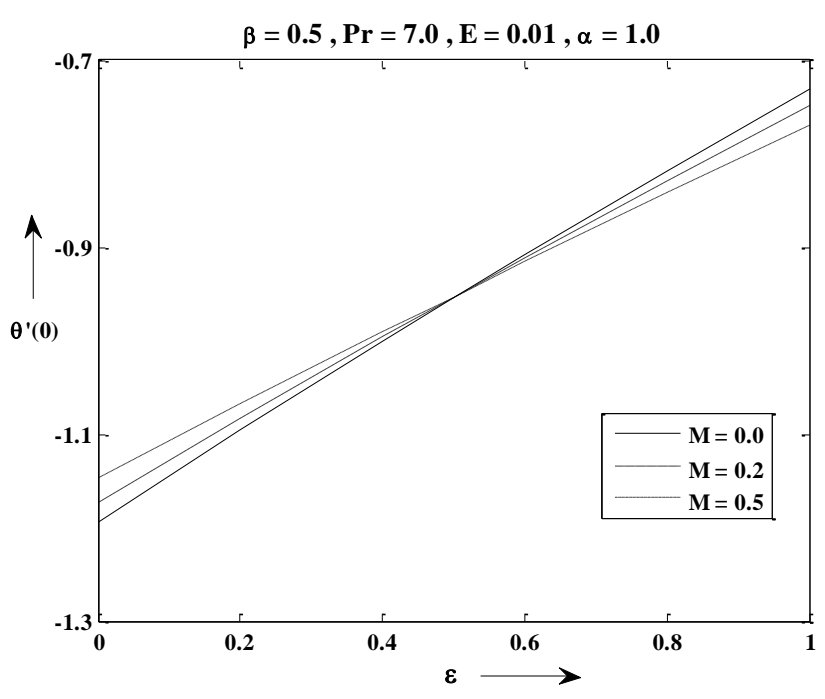

Fig. 12 The rate of heat transfer for different $M$.

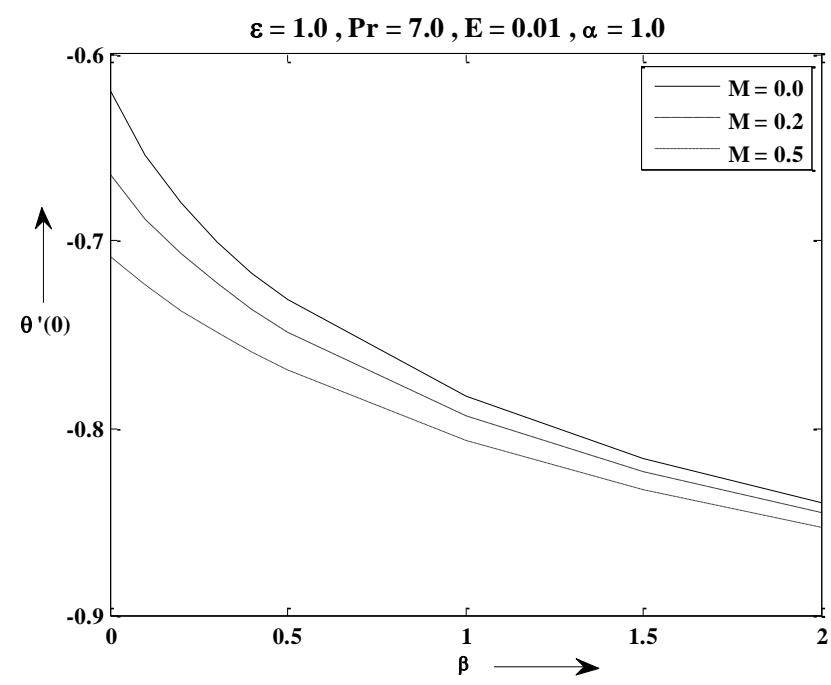

Fig. 14 The rate of heat transfer against $\beta$ for different $M$ 


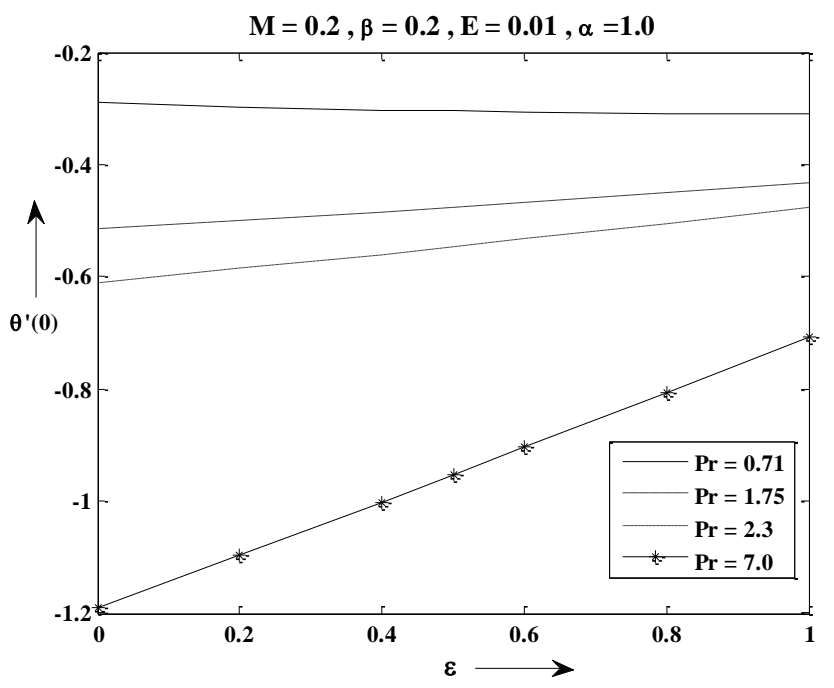

Fig. 15 The rate of heat transfer against $\varepsilon$ for different $\operatorname{Pr}$. 